\title{
8
}

Georgia Hardavella ${ }^{1,2}$, Ane Aamli-Gaagnat ${ }^{3}$, Armin Frille ${ }^{4}$,

[1]

'Dept of Respiratory Medicine, King's College Hospital, NHS Foundation Trust, London, UK. '2Dept of Respiratory

Medicine and Allergy, King's College, London, UK. ${ }^{3}$ Dept of Clinical Science, University of Bergen, Bergen,

Norway. ${ }^{4}$ Dept of Respiratory Medicine, University of Leipzig, Leipzig, Germany. ${ }^{5}$ National Heart and Lung

Institute, Imperial College London, London, UK. 'European Respiratory Society, Lausanne, Switzerland.

${ }^{7}$ European Lung Foundation, Sheffield, UK.

\section{Top tips to deal with challenging situations: doctor-patient interactions}

\section{Doing science}

Raise your words, not your voice. It is rain that grows flowers, not thunder.

Rumi

Interactions between patients and medical practitioners can sometimes be challenging. We have all had consultations where the interaction was not optimal, either as medical practitioners or as a patient ourselves. Neither normally wishes to cause a difficult situation but common misunderstandings, by both groups, often result in such an occurrence. Communication and listening skills are essential for every consultation but in particular, for situations where the interaction may become difficult.

In this article, we will discuss what may make a consultation difficult and what outcomes this could lead to, and provide some suggestions to help both you and your patient.

\section{What is a challenging interaction and how might it be perceived?}

Many different challenging interactions occur daily. These challenging interactions may arise due to discrepancies in expectation, perception and/or communication between the patient and medical practitioner, and could be caused by the doctor, by the patient or by both. We have outlined a list of potential scenarios in table 1 and discuss how these might be perceived from both a healthcare professional and patient perspective.

Examples of scenarios include when a doctor:

- informs the patient of bad news without ensuring that this is done in an appropriate setting (e.g. breaking bad news in a busy corridor at the accident and emergency department in the presence of medical students and other patients that are observing);

- delivers difficult news (e.g. a life-changing diagnosis) without showing empathy or ensuring there is appropriate support available for the patient (e.g. counselling services or caregivers/ family members around);

- during a consultation, uses poor nonverbal communication (e.g. no eye contact with the patient, instead focussing solely on the computer screen or notes; stance; gestures; or tone of voice); or

- speaks ambiguously, not explaining, in plain language, long-term management plans, or the importance or implications of diagnosis.
Cite as: Hardavella G, Aamli-Gaagnat A, Frille A, et al. Top tips to deal with challenging situations: doctor-patient interactions. Breathe 2017; 13: 129-135 
Table 1 Most common real-life scenarios where an interaction with a patient can be challenging

The patient presents a long list of symptoms

The patient feels they are not being listened to

There is no diagnosis despite thorough work-ups

Drug dose decrease

Delivering bad news

Noncompliance

Alternatively, these scenarios may arise when a patient:

- has done research online about their ailments and is convinced by their findings of a conclusion, and demands certain investigations/treatments;

- feels that they are not being listened to and might become frustrated, or threaten legal action or social media involvement;

- does not accept the doctor's diagnosis or test results and demands a second opinion;

- has symptoms affecting their quality of life but no diagnosis despite thorough work-ups by various medical teams, which can lead to frustration or a lack of trust in medical professionals;

- will not follow the suggested treatment but continues to attend consultations with deteriorating health (e.g. a heavy smoker with severe asthma who does not stop smoking and believes that their inhalers do not work); or

- will focus on what went wrong rather than what is the best way to progress things.

\section{Factors contributing to a challenging interaction}

To prevent and resolve challenging interactions, one needs to consider factors that might contribute to these situations. Two important factors are the local healthcare setting in which the interactions take place, and the variation in clinical practice between regions and countries. In particular, the majority of healthcare settings are overworked and overstretched to meet demand, and this continuously affects interactions. Insufficient time for consultation or interaction with patients plays an important role, as healthcare system pressures are increasing patient numbers and expectations, against a background of cost-cutting. Foremost, it is important to bear in mind that both patients and healthcare practitioners want a positive interaction to ensure the best possible health outcome, as time spent in consultations is valuable for both parties. Figure 1 summarises several other important contributing factors.

\section{The patient}

Each patient has their own medical and psychosocial history that understandably will affect their behaviour. Patients will walk into your clinic with a set of beliefs and expectations affected by their personality and the severity of their symptoms, and the implications of this for their quality of life. They may also have had negative experiences and previous disappointments within the healthcare system that may be challenging to overcome

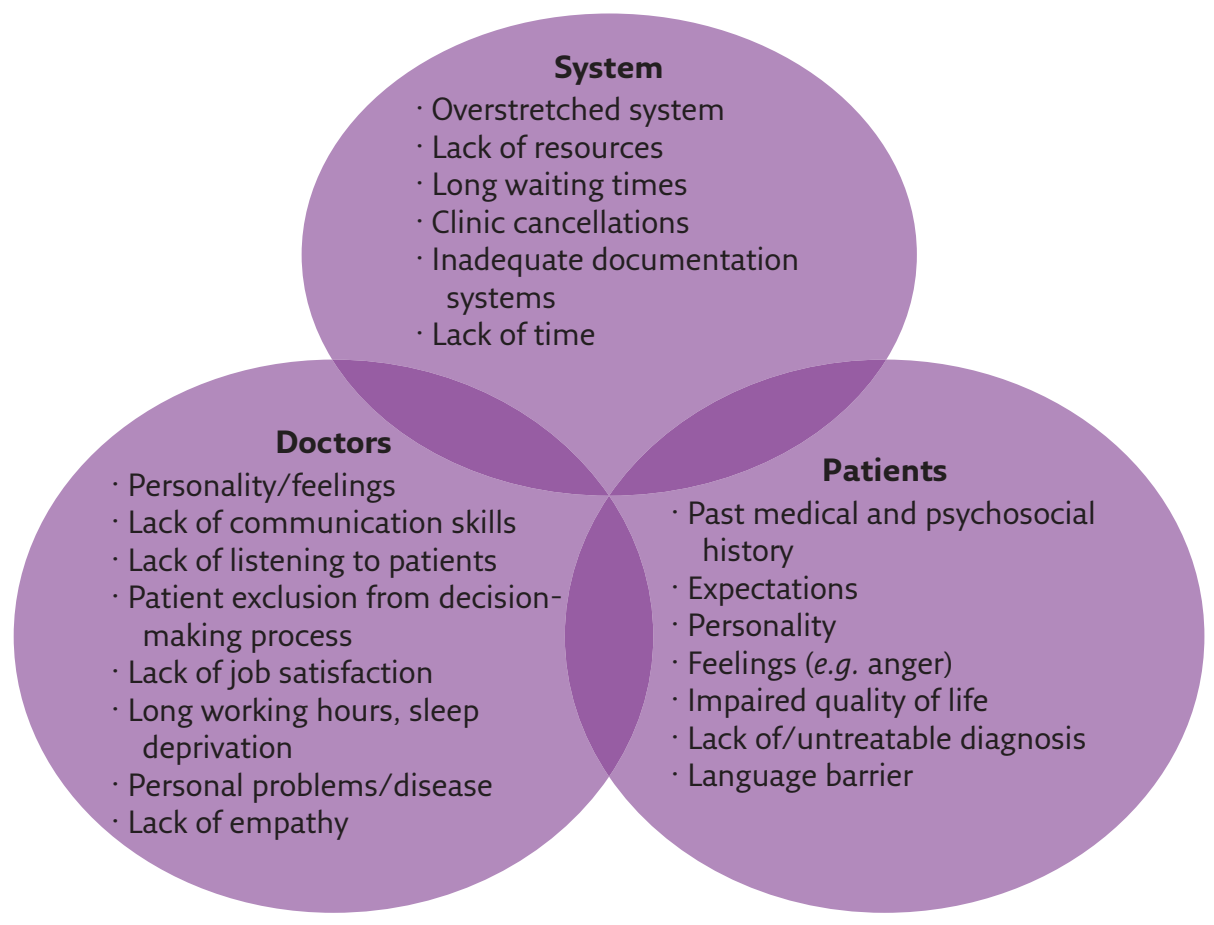

Figure 1 Factors contributing to a challenging interaction. 
and may generate some mistrust. They may feel that their illness is beyond their personal control, which can make them dependent on others' help, particularly their healthcare professional. Such circumstances can, understandably, make a patient feel anxious, worried, hopeless and uncertain about their health, which can be displayed as tension and negative reactions towards the healthcare professional.

With increasing advances in medical research, expectations of the healthcare system and in healthcare practitioners have also increased. Patients can have very high expectations and trust in the system, and when it appears that their condition is a medical "dead end" or that their prognosis cannot be determined with precision due to the nature of the disease, it can be very upsetting. Language barriers, cultural diversity and their previous interactions with professionals or authority figures can also contribute to and affect interactions, and lead to misunderstandings. Moreover, patients may also have other considerations to make, for example, if their diagnosis may impact on other commitments (professional, caring responsibilities, etc.). Patients often work, may care for children or parents, or have other commitments that may be impacted by the diagnosis or may have impacted on the timeframe in which they seek help, all of which will be going through their mind. Being defined by their diagnosis and labelled as "a patient" is not, and should not be, the only thing in their lives.

\section{The healthcare practitioner}

There is a wide variability in the development of appropriate communication skills among European healthcare practitioners and this has been a challenge. Communication skills courses or training are not included in the specialist curriculum in all European Union (EU) countries, nor are they included in the essential qualifications for specialist post applications.

A lack of communication skills training can result in:

- inappropriate choice of words and phrases, perhaps due to assumptions being made about the patient's level of health literacy or understanding of human biology;

- lack of planned structure in delivering difficult news (e.g. scattered information confusing patients or no clear plan at all);

- inappropriate choice of setting to deliver difficult news;

- lack of options offered to the patient;

- not involving the patient in the decision-making process (e.g. treatment decisions taken without involving them and without addressing their needs and wishes);

- rushing the patient to agree to a proposed treatment plan;
- rushing the consultation due to other pressures; or

- not referring the patient to appropriate support services/resources (e.g. counselling, palliative care, support groups and quality trusted information).

Bad news may be broken in a nonempathetic way, messages may be given to the nurses over the patient's head while interrupting the consultation, difficult words may be used that the patient does not understand, and the patient may feel excluded from conversations with almost no concern showed for their feelings and emotions. Often, what is everyday routine clinical data to the healthcare practitioner may be completely unfamiliar to the patient, giving the impression that the clinician is cold and unsympathetic to the individual's emotions as they try to come to terms with the diagnosis and its implications.

Overstretched clinic time may result in doctors not having time to actually listen to the patient's concerns. What is the patient actually afraid of? What do they want to know? What are their experiences? These are questions that will be overlooked due to lack of time. Insufficient time further impacts the consultation as there is not time for the patient to verbalise, and for the doctor to appreciate, the valuable contribution that the patient brings in having the lived experience of the condition, especially if this is a rare disease.

In a complex clinical case, doctors may seem so preoccupied with finding the solution to the clinical problem that it is sometimes easy to forget that the patient might be overwhelmed by anxiety, frustration and negative emotions, and require re-assurance to feel safe, at ease and trust in the doctor.

Healthcare setting (either outpatient clinic or wards) is a familiar setting for doctors to have difficult conversations, whereas for patients, it can be uncomfortable and sometimes awkward, especially if they are at the point of receiving their diagnosis.

In addition, a doctor's emotions may get the better of them or their behaviour might be affected by a lack of sleep, hunger, their own health status, lack of job satisfaction or other concerns. Finally, the doctor's approach and communication style will influence their interactions and could have serious adverse effects on the patient (e.g. if the healthcare practitioner is arrogant or impatient and believes they don't have a responsibility to discuss the situation with the patient or explain the condition in terms the patient could understand).

It's important for doctors to recognise that some patients may be intimidated and perceive inequality in the doctor-patient relationship, which can be exacerbated by doctors acting in a way that is perceived by the patient as condescending or patronising. All this can be remedied with appropriate training and relevant professional development. 


\section{The system}

Dysfunctional healthcare systems can only add to the tension between patients and doctors. Simple things like long waiting times in the clinic, consecutive unjustified cancellations, or delays to previous appointments or investigations; essentially, anything that may have gone wrong in the patient pathway can potentially lead to a challenging interaction between patients and doctors. Doctors are probably the first person patients will spend some time with after something has gone wrong and therefore they will hear the patient's immediate frustrations first hand.

Lack of resources in terms of staffing levels or of maintaining patient privacy and dignity during consultation is another contributing factor; for example, during a consultation there may be several doctors or nurses moving in and out of the room that distract attention and may affect dignity and privacy.

A lack of centralised documentation systems can sometimes lead to asking the patient to repeat the same information over and over again, and consequently dedicating less time to actually managing the clinical case and addressing the patient's needs. Constant repetition for every new doctor may cause the patient frustration, while it is difficult for the doctor to know what the patient already understands.

\section{Potential effects of a challenging interaction}

Above all, it should be acknowledged that patients want a positive interaction with their doctor.

Table 2 Potential implications of a challenging interaction

Patient
Anxiety
Concern
Frustration
Dissatisfaction
Vulnerability
Loss of trust in the doctor-patient relationship
Doctor
Stress, anxiety and anger
Helplessness
Dislike of the patient
Use of avoidance strategies (e.g. discharge)
System
Misuse of more resources
Appointment with another doctor for a second opinion
Increased attendance at the emergency department

In reality, a challenging interaction between patients and doctors should be considered within the healthcare system in which it occurs. Patients seek professional help because they are in pain or are concerned.

When the three factors of the patient, the doctor and the system interact, a particularly difficult situation can arise. Figure 1 summarises the most important contributing factors, which are outlined below. We all respond differently when in a challenging situation but our behaviour or response could have serious detrimental effects (table 2).

\section{Patients}

Patients can be overwhelmed by a variety of beliefs and emotions: frustration, feeling they have little to no control over their diagnosis and health condition, uncertainty over the course of their treatment and prognosis, fear, worries, and overall dissatisfaction with the healthcare system. Communication between the patient and medical professionals may then be prejudiced and result in the patient losing trust in the doctor. This can be further affected by the implications of the condition itself on the patient's psychology.

Due to the combination of all this, patients can feel they are not heard and consequently feel more vulnerable. They may have already arrived at the clinic in a state of some anxiety after various tests, investigations or previous appointments. They may be anticipating bad news or may be reluctant to consider various treatment options, believing these may disrupt theirs or their loved ones' quality of life. They may have had had previous poor experiences of hospital or healthcare settings and may fear that raising concerns or asking questions could delay or otherwise impact on their treatment. Their culture or upbringing may have led them to believe they should not ever question somebody in authority even if they have lots of questions. A clinician rushing through an appointment may be perceived as "harsh" or less considerate than one who takes the time to listen to the patient's concerns.

\section{Healthcare practitioners}

A challenging interaction for a medical professional already overstretched by the healthcare system may increase levels of stress, anxiety and anger, which in turn will impact on performance and communication.

Generally, physicians tend to feel helpless after a challenging interaction with a patient, and may be unsure about how to take things forward or whom to consult for advice. As previously stated, in most EU countries, there is a lack of training in how to manage these cases and a possible response might be to move the patient to another colleague (i.e. avoidance). 


\section{System}

The potential effects to the patient and doctor will put more pressure on the system, as they may result in overuse of resources. This means that the patient will either try the "doctor shopping" approach, i.e. seeing several different doctors for the same issue and trying to collect different opinions, or inappropriately attending the accident and emergency department frequently trying to find a solution to a nonacute issue. Sometimes, patients adopt both approaches, which can overstretch healthcare systems in terms of capacity and costs.

\section{Managing a challenging interaction}

The optimal approach in dealing with a challenging interaction is to prevent it. If that is not possible, then it is best to create the conditions for dealing with a difficult situation in a manner that is open and safe for all, and to develop the skills of active listening and effective communication (table 3).

\section{Plan your interaction in advance}

- Think in advance how best to deliver that news to that particular patient, and structure your thoughts

Choose appropriate words that will not offend or be perceived negatively. It is important to break down information into small pieces that are easy to understand and to ensure the patient has a clear understanding before progressing the conversation. Asking patients to reiterate and confirm halfway through the conversation, and summarise at the end, is always efficient, and ensures both parties share the same information and action plans.

- Do not under-communicate the difficulties that occur with the disease

Remember that it is far better for patients to be prepared and to participate in the treatment decision-making process than to be kept in the dark or, even worse, be undermined. Try to create a positive "teamwork" with the patient.
- Consider the role of the patient's partner or carer during the consultation.

This may be the patient's spouse, parent or friend who can help support the patient during the consultation. They may also have their own questions or concerns about the condition, which should be addressed. The inclusion of a partner or carer is essential, especially in a "bad news" conversation. Two pairs of ears are better than one, especially when the information being received is negative, unexpected and/or difficult to understand. In such situations, it can be hard for the patient to take in. For such conversations, find out when the family member is available to be party to the conversation

- Ensure you deliver the news in an appropriate setting, check they can hear you and ensure patient consent is obtained prior to having multiple people observing your consultation (e.g. students)

\section{Pay attention to your nonverbal communication}

Nonverbal communication is equally important as the actual words a clinician uses during their interaction with the patient. Body posture, gestures and eye contact can all combine with verbal communication to facilitate a meaningful positive communication with your patient.

\section{Provide ways to access further information and support}

Some patients may not take in all of the information you provide up front, particularly if they have received a new diagnosis. Providing your contact details, such as your e-mail address and telephone number, may allow them to ask you questions in their own time, after the consultation has finished. Avoid telling patients not to read anything on the internet about their condition, but rather, consider ways in which you can provide access to additional information and support, including sign-posting to

Table 3 Tips on managing difficult interactions

Plan your interaction in advance

Pay attention to nonverbal communication

Discuss with colleagues and do not hesitate to seek additional training should this be required

Look for signs of anger or distress

Ensure safety and maintain control

Create bridges of communication and trust

Explain the difficulty and try to find common ground

Help your patient get emotional control

Focus on highlighting solutions and resolve areas of disagreement 
counselling, support services and patient support groups. Patient information resources published by your organisation are also encouraged.

\section{Keep the initial information simple and try not to use too advanced medical language}

Reassure patients that it is a good idea for them (or their carer/partner) to write things down, whether at the time of a consultation or a list of questions in advance of a consultation.

\section{Discuss with colleagues and do not hesitate to seek additional training should this be required}

What may be a challenging interaction for one person may differ from someone else. The human factor significantly contributes to the different perceptions further complicated by different experiences and subsequently different comfort levels in dealing with different personality types and situations. There is no "one size fits all" approach. You need to be open to learning and developing your practice, and discuss with colleagues or your mentor as this will provide you with valuable advice. On a similar note, never hesitate to seek additional training to further develop your communication skills (either online, face to face or at a professional development workshop). In a scientific, evidencebased, clinical setting, it may seem unfamiliar to develop so called "soft" or interpersonal skills but the techniques learnt will be just as valuable when communicating with colleagues and patients, and building relationships generally.

\section{Is this becoming a difficult situation?}

Look for signs of anger or distress, an increase in speed of speech, or a change in behaviour or body language. This may indicate that the patient is uncomfortable with the conversation or procedure. Steer the conversation away from the topic and address it when the patient is more comfortable discussing it or consider whether the procedure is immediately necessary.

\section{Create bridges of communication and trust}

Another fundamental requirement for a positive interaction to occur is ensuring that the patient's psychological safety is ensured. This is particularly relevant for taking small risks when interacting with the patient while, at the same time, facing uncertainty or ambiguity. The solution here is to focus on creating favourable conditions in which any interpersonal risks between you and the patient are kept to a minimum. For instance, reassure the patient that they can feel safe and communicate openly with you in order to establish trust and ensure there is sufficient time scheduled for the consultation, so that you are not rushed.

\section{Explain the difficulty and try to find common ground}

Try to put the focus on the "elephant in the room", i.e. verbalise the problem in a kind yet clear manner and find some common ground with the patient. Establishing common ground is a key point in reducing any tension that may have arisen. The patient needs to have confidence and actually recognise that you are listening to them. For example, you can show understanding towards the patient's anxieties and worries, and reflect this understanding back to the patient. This shows empathy, and may help the patient feel more comfortable, let off some steam and voice any underlying problem or concern that contributed to the challenging interaction. On some occasions during these "de-escalation" conversations you may realise that there is an alternative explanation to the patient's feelings and this may have increased their anxiety or fear.

\section{Help your patient get emotional control}

Feeling helpless and hopeless is a common challenge encountered by a patient facing a difficult health condition. Imagine being a patient yourself. Not being in control can trigger negative emotions and can make communication difficult. Empathy and effective listening can help with this. Also, keeping the patient informed and involving them in the decision-making process is the basis for giving them a sense of control. Bear in mind the psychological impact of your conversations with the patient and the possible symptoms it might cause, particularly in respiratory patients. For example, many of our patients develop problems with anxiety, panic attacks and hyperventilation, which can prove more debilitating than some of the other symptoms of their condition.

\section{Focus on highlighting solutions if there are areas of disagreement}

After summarising the situation and hearing opinions from both sides, give the patient two or three options with balanced information; this changes the focus from any miscommunication or argument to action, and helps to redirect the patient to a solution-focused path. Allow time for questions and discussion with the patient. The patient should feel valued and involved in the decision-making process. 


\section{Ask the patient how the consultation went.}

We must admit that most of us forget to do this. Use open questions along the lines of "How do you think your consultation was today?". Take time to listen and, where necessary, clarify.

\section{Get some time to reflect}

After any challenging interaction, it is important to reflect on what happened and identify what could be improved. How did your actions contribute to the situation and what could you have done differently? Think about difficult people and situations as your teachers, not your enemies. How will you take that learning forward for next time?
This thought process will help you in future challenging situations and provide better insight as to how to manage similar cases in the future. It is always useful to discuss this with peers/colleagues to get some feedback and update your supervisor or head of the department.

Regardless of the outcome, your personality, patient characteristics and challenges in the healthcare system, try to stay in line with your mission to deliver optimal medical care to all your patients.

\section{Acknowledgements}

Many thanks to Gill Hollis, Lisbeth Høva and Janette Rawlinson, who kindly provided feedback and gave us patients' perspective on our manuscript.

\section{Conflict of interest}

A. Niculescu is an employee of the European Respiratory Society and P. Powell is an employee of the European Lung Foundation.

\section{Suggested reading}

1. Philip J, Kissane DW. Responding to difficult emotions. In: Kissane DW, Bultz B, Butow P, et al. Handbook of Communication in Oncology an Palliative Care. New York Oxford University Press, 2010; pp. 135-146.

2. Jackson JL, Kroenke K. Difficult patient encounters in the ambulatory clinic: clinical predictors and outcomes. Arch Intern Med 1999; 159: 1069-1075.

3. Kreger J. When your patients are in mourning. FPM. 2003; 10 : 49-50.

4. Epstein RM. Mindful Practice. JAMA 1999; 282: 833-839.
5. Edmondson AC. Learning from failure in health care: frequent opportunities, pervasive barriers. Qual Safety Health Care 2004; 13: Suppl. 2, ii3-ii9.

6. Bramson RM. Coping with difficult people. Garden City, Anchor Press/Doubleday, 1981.

7. European Respiratory Society. E-Learning resources: How to communicate effectively with patients. www.ers-education. org/events/courses/how-to-communicate-effectively-withpatients.aspx Date last accessed: March 23, 2017. 